Pacific Journal of Mathematics

ON THE ZEROS OF SOLUTIONS OF SOME LINEAR 


\title{
ON THE ZEROS OF SOLUTIONS OF SOME LINEAR COMPLEX DIFFERENTIAL EQUATIONS
}

\author{
DAVID V. V. WEND
}

Introduction. In this paper Green's function methods are used to investigate the distribution on the real axis of zeros of solutions of the complex differential equations

$$
\left(p(x) y^{\prime}\right)^{\prime}+f(x) y=0
$$

and

$$
y^{\prime \prime \prime}+f(x) y=0 .
$$

In both cases the coefficient $f(x)$ is assumed to be complex-valued and continuous on a half-line $I: x_{0} \leq x<\infty$, while $p(x)$ in equation (1) is assumed to belong to a special class of complex-valued functions to be defined in Section I.

Equation (1) or equation (2) is said to be nonoscillatory on a set $E$ if no nontrivial solution has an infinite number of zeros in $E$. In what follows a solution shall mean a nontrivial solution. Suppose in equation (1) $x$ is a complex variable and $p(x)$ and $f(x)$ are analytic in a simplyconnected region $R$. Consider the well known Green's function $g(x, s)$ for the differential system

$$
\left(p(x) y^{\prime}\right)^{\prime}=0, \quad y(a)=y(b)=0,
$$

where $a$ and $b$ are distinct points of $R^{1}$. If $a$ and $b$ are zeros of a solution of equation (1), then

$$
1 \leq \int_{a}^{b}|g(x, s)||f(x)||d x|,
$$

where the integral is taken along a path $C$ in $R$ and $s$ is an interior point of $C$. Starting with this inequality and imposing various bounds on $|f(x)|$, Z. Nehari [7] and P. R. Beesack [3] have obtained nonoscillation theorems for $y^{\prime \prime}+f(x) y=0$ in various regions of the complex plane where $f(x)$ is analytic. By the same methods the author [2] has extended some of these theorems and obtained similar results for equation (1). The methods used in this paper are essentially those employed in the sources mentioned above. However, by restricting the independent variable to the real axis the condition of analyticity is relaxed and

Received May 21, 1959. This research was sponsored by the Office of Ordnance Research under Contract DA-04-495-ORD-1088. Presented to the American Mathematical Society November, 1958.

1 Sufficient conditions for the existence of $g(x, s)$ are given in [2, p 15]. 
upper bounds on the number of zeros of a solution on a given interval are obtained not only for equation (1) but also for the third order equation (2).

1. A nonoscillation theorem. In this section we will consider equation (1). It will be assumed that $p(x)$ is continuous and different from zero on $I$. In order to make use of Green's function we wish to have the system (3) incompatible, i.e., possess no (nontrivial) solution on $I$.

If $p(x)$ is allowed to be complex-valued on $I$, then the system (3) may be compatible. For example the system $\left(e^{-i x} y^{\prime}\right)^{\prime}=0, y(2 m \pi)=0$, $y(2 n \pi)=0, m$ and $n$ distinct positive integers, has the nontrivial solution $y(x)=e^{i x}-1$ on $I: 0 \leq x<\infty$. In order to avoid such examples and also to be able to make use of certain estimates of Green's function, only a restricted class of functions $p(x)$ will be considered.

Definition. Let $G(I)$ denote the class of all complex-valued, continuous and non-zero functions $p(x)$ defined on $I: x_{0} \leq x<\infty$ which possess the further property that for any three numbers $a, b$ and $c$ such that $x_{0} \leq a<b<c<\infty$,

$$
\left\{\begin{array}{l}
\text { (a) }\left|\int_{a}^{b} \frac{d x}{p(x)}\right|<\left|\int_{a}^{c} \frac{d x}{p(x)}\right| \\
\text { (b) }\left|\int_{b}^{c} \frac{d x}{p(x)}\right|<\left|\int_{a}^{c} \frac{d x}{p(x)}\right|
\end{array}\right. \text {. }
$$

Note. The class $G(I)$ contains the functions $p(x)>0$ which are continuous on $I$.

An interesting subclass of $G(I)$ is the collection of complex-valued functions $p(x)$ in $G(I)$ which possess the additional property that if

$$
\varphi(x)=\int_{x_{0}}^{x} \frac{d t}{p(t)}=u(x)+i v(x),
$$

then

$$
\left(\frac{d u}{d x}\right)^{2}+\left(\frac{d v}{d x}\right)^{2} \neq 0 \text { on } I
$$

and for any $x^{\prime}, x^{\prime \prime}$ in $I, \theta^{\prime}=\left.\arctan (d v / d x / d u / d x)\right|_{x=x^{\prime}}$ and

$$
\theta^{\prime \prime}=\left.\arctan \left(\frac{d v / d x}{d u / d x}\right)\right|_{x=x^{\prime \prime}}
$$

can be chosen so that $\left|\theta^{\prime}-\theta^{\prime \prime}\right|<\pi / 2$. In effect, the image curve of $I$ under $\varphi(x)$ cannot change direction by more than $\pi / 2$. 
Suppose $p(x) \in G(I)$. Then the differential system (3) is incompatible. Therefore the Green's function for this system exists, and it has the explicit form

$$
g(x, s)=\left\{\begin{array}{l}
\int_{a}^{x} \frac{d t}{p(t)} \int_{s}^{b} \frac{d t}{p(t)} / \int_{a}^{b} \frac{d t}{p(t)}, a \leq x \leq s \\
\int_{a}^{s} \frac{d t}{p(t)} \int_{x}^{b} \frac{d t}{p(t)} \mid \int_{a}^{b} \frac{d t}{p(t)}, s \leq x \leq b
\end{array}\right\},
$$

$a<s<b$.

Since $p(x) \in G(I)$, the inequalities (4) are satisfied and these inequalities together with the above expressions for $g(x, s)$ show that

$$
|g(x, s)|<\left\{\begin{array}{ll}
\text { (a) } & \int_{a}^{b} \frac{d t}{|p(t)|} \\
\text { (b) } & \int_{a}^{x} \frac{d t}{|p(t)|} \\
\text { (c) } & \int_{x}^{b} \frac{d t}{|p(t)|}
\end{array}\right\},
$$

$x \neq a, b$ and $s \neq a, b$.

If $y(x)$ is a nontrivial solution of equation (1) on the interval $a \leq x \leq b$ such that $y(a)=y(b)=0$, then the inequalities (4) imply $f(x)$ is not identically zero on $a \leq x \leq b$ and

$$
y(x)=\int_{a}^{b} g(x, s) y(s) f(s) d s .
$$

If $x$ is chosen so that $|y(x)|$ is a maximum on the interval $a \leq x \leq b$, then (following Z. Nehari [7]):

$$
1<\int_{a}^{b}|g(x, s)||f(s)| d s .
$$

As a consequence of inequalities (6) and (7),

$$
1< \begin{cases}\text { (a) } & \int_{a}^{b}|f(x)| d x \int_{a}^{b} \frac{d x}{|p(x)|} \\ \text { (b) } & \int_{a}^{b}|f(x)|\left(\int_{a}^{x} \frac{d t}{|p(t)|}\right) d x . \\ \text { (c) } & \int_{a}^{b}|f(x)|\left(\int_{x}^{b} \frac{d t}{|p(t)|}\right) d x\end{cases}
$$

Theorem 1. Suppose $p(x) \in G(I)$, and $a_{1}<a_{2} \cdots<a_{n}$ are $n$ consecutive zeros of a solution of $\left(p(x) y^{\prime}\right)^{\prime}+f(x) y=0, a_{1} \geq x_{0}$. Then $a_{n}$ 
must satisfy the inequalities

$$
\begin{cases}\text { (a) } & n-1<\int_{x_{0}}^{a_{n}}|f(x)| d x \int_{x_{0}}^{a_{n}} \frac{d x}{|p(x)|} \\ \text { (b) } & n-1<\int_{x_{0}}^{a_{n}}|f(x)|\left(\int_{x_{0}}^{x} \frac{d t}{|p(t)|}\right) d x . \\ \text { (c) } n-1<\int_{x_{0}}^{a_{n}}|f(x)|\left(\int_{x}^{a_{n}} \frac{d t}{|p(t)|}\right) d x\end{cases}
$$

Proof. Since $p(x) \in G(I)$, the inequalities (8) are satisfied for $a$ and $b$ zeros of a solution of equation (1). From inequality (8a)

$$
1<\int_{a_{j}}^{a_{j+1}}|f(x)| \int_{a_{j}}^{a_{j+1}} \frac{d x}{|p(x)|}, \quad j=1,2, \cdots, n-1 .
$$

Adding these $n-1$ inequalities,

$$
n-1<\sum_{j=1}^{n-1} \int_{a_{j}}^{a_{j+1}}|f(x)| d x \int_{a_{j}}^{a_{j+1}} \frac{d x}{|p(x)|} \leq \int_{x_{0}}^{a_{n}}|f(x)| d x \int_{x_{0}}^{a_{n}} \frac{d x}{|p(x)|},
$$

giving the inequality (9a). The inequalities $(9 \mathrm{~b})$ and $(9 \mathrm{c})$ follow in similar fashion from the inequalities (8b) and (8c), respectively.

The following theorem is an immediate corollary of Theorem 1 .

THEOREM 2. Nonoscillation theorem. Suppose $p(x) \in G(I)$ and

$$
\begin{aligned}
& L=\int_{x_{0}}^{\infty}|f(x)|\left(\int_{x_{0}}^{x} \frac{d t}{|p(t)|}\right) d x, \\
& M=\int_{x_{0}}^{\infty}|f(x)|\left(\int_{x}^{\infty} \frac{d t}{|p(t)|}\right) d x,
\end{aligned}
$$

where $L$ and $M$ many assume the value $+\infty$. Then $\left(p(x) y^{\prime}\right)^{\prime}+f(x) y=0$ is nonoscillatory on $I$ if either $L$ or $M$ is finite, and if either $L$ or $M$ is less than 1 , then equation (1) is disconjugate on $I$, i.e., no solution has more than one zero on $I$.

In the case $f(x)$ and $p(x)$ are real, the tests in Theorem 2 compare with known criteria, for example those of W. Leighton [5, Corollary 4.2], E. Hille [4, p. 238], R. A. Moore [6, Theorems 3, 4 and 7 Corollary 1] and R. L. Potter [8, Theorem 4.2].

2. An example. The substitution $y=v / \sqrt{p}$ transforms equation (1) into the normal form

$$
v^{\prime \prime}+F(x) v=0
$$

where 


$$
F(x)=\frac{f}{p}+\frac{1}{4}\left(\frac{p^{\prime}}{p}\right)^{2}-\frac{1}{2}\left(\frac{p^{\prime \prime}}{p}\right),
$$

and equation (1) is nonoscillatory if and only if equation (10) is nonoscillatory. With $p(x) \equiv 1$, the constant $M$ in Theorem 2 is infinite, while the nonoscillation condition

$$
L=\int_{x_{0}}^{\infty}|f(x)|\left(\int_{x_{0}}^{x} \frac{d t}{|p(t)|}\right) d x<\infty
$$

is equivalent to

$$
\int_{x_{0}}^{\infty} x|F(x)| d x<\infty .
$$

In the following differential equation the integral in (12) is infinite, hence fails to show nonoscillation, while in (11) $L<2$, showing that no solution of the equation can have more than two zeros on $I$. Let

$$
\left(\frac{x^{2}}{2-\sin \log x} y^{\prime}\right)^{\prime}+\frac{1}{(x+i)^{2}} y=0, x_{0}=1 \text {. }
$$

Since $p(x)=x^{2} /(2-\sin \log x)>0$ on $I, p(x) \in G(I)$, and it is easily estimated that

$$
L=\int_{1}^{\infty} \frac{1}{|x+i|^{2}}\left(\int_{1}^{x} \frac{2-\sin \log t}{t^{2}} d t\right) d x<\frac{3}{2} .
$$

For equation (13)

$$
F(x)=\frac{2-\sin \log x}{x^{2}(x+i)^{2}}+\frac{1}{4 x^{2}}\left(\frac{-3 \cos ^{2} \log x}{(2-\sin \log x)^{2}}+\frac{2 \sin \log x-2 \cos \log x}{2-\sin \log x}\right),
$$

and easy estimations give

$$
\begin{aligned}
\int_{1}^{\infty} x|F(x)| d x & \geq \int_{1}^{\infty} \frac{1}{4 x}\left|\frac{2 \sin \log x-2 \cos \log x}{2-\sin \log x}-\frac{3 \cos ^{2} \log x}{(2-\sin \log x)^{2}}\right| d x \\
& -\int_{1}^{\infty} \frac{2-\sin \log x}{x\left(x^{2}+1\right)} d x=I_{1}-I_{2},
\end{aligned}
$$

where $0<I_{2}<3 / 4$. Letting $t=\log x$ in $I_{1}$,

$$
\begin{aligned}
I_{1} & \geq \frac{1}{36} \int_{0}^{\infty}\left|1+(\cos t-\sin t)^{2}+\cos ^{2} t-4(\sin t-\cos t)\right| d t \\
& =\frac{1}{36} \int_{0}^{\infty}|k(t)| d t .
\end{aligned}
$$

From the graph of $k(t), k(t)>1$ for $0<t<\pi / 4$ and $5 \pi / 4<t<2 \pi$, 
while $k(t)<-1$ for $3 \pi / 4<t<\pi$, so $|k(t)|>1$ for intervals of length $5 \pi / 4$ out of each interval of length $2 \pi$ on $0 \leq t<\infty$. Therefore $I_{1}=\infty$, so $\int_{1}^{\infty} x|F(x)| d x=\infty$.

3. Distribution of zeros. Suppose the upper limits of the integrals on the right in the inequalities (9) are considered as continuous variables and $f(x)$ is not identically zero on any subinterval of $I$. Then in each case the integral is a strictly monotone increasing function of the upper limit and there exists at most one value of the upper limit for which equality will hold. If $x_{1}$ is such a value, then no solution of equation (1) can have more than $n$ zeros on the interval $x_{0} \leq x \leq x_{1}$. Since $a_{n} \geq x_{1}$, the value $x_{1}$ also gives a lower bound on the magnitude of the $n$th consecutive zero on $I$ of any solution of equation (1).

Adapting the notation used in [6], let $N\left(x_{1}, x_{2}\right)$ be the maximum number of zeros any solution of equation (1) may have on the interval $x_{1} \leq x \leq x_{2}$. Since in the complex case there is often no zero separation theorem, the number $N\left(x_{1}, x_{2}\right)$ merely puts an upper bound on the number of zeros a particular solution may have. See [1, Theorem 1.2].

As an application of the above discussion we give the following theorem:

Theorem 3. Suppose $a_{1}<a_{2}<\cdots<a_{n}, 1 \leq x_{0} \leq a_{1}$, are $n$ consecutive zeros of a solution of

$$
\left(x^{\sigma} y^{\prime}\right)^{\prime}+f(x) y=0
$$

and $H=\int_{x_{0}}^{\infty}|f(x)| d x<\infty$. If $0 \leq \sigma<1$, then

$$
[1+(n-1)(1-\sigma) / H]^{1 /(1-\sigma)}=x_{1}<a_{n}
$$

and $N\left(x_{0}, x_{1}\right)<n$. If $\sigma=1$, then

$$
\exp \left[\frac{n-1}{H}\right]=x_{2}<a_{n}
$$

and $N\left(x_{0}, x_{2}\right)<n$.

Proof. Inequality (14) follows from inequality (9a). Inequality (15) may be obtained from inequality (14) by letting $\sigma \rightarrow 1$ or directly from inequality $(9 b)$.

Other lower bounds on the magnitude of the zeros of solutions of equation (1) can be obtained by considering the maximum value of $|g(x, s)|$ on $a \leq x \leq b, a<s<b$. We assume $p(x)>0$ and continuous on $I$. From the expressions for $g(x, s)$ given in (5) it can be shown 
that the maximum value of $|g(x, s)|$ occurs when $x=s$ and $s$ satisfies the equation

$$
\int_{a}^{s} \frac{d x}{p(x)}=\int_{s}^{b} \frac{d x}{p(x)} \cdot \quad(\text { Compare [3, p. 231].) }
$$

As an illustration of this result we give the following theorem:

THEOREM 4. Suppose $a_{1}<a_{2}<\cdots<a_{n}, 0 \leq x_{0} \leq a_{1}$, are $n$ consecutive zeros of a solution of equation (1) and $H=\int_{x_{0}}^{\infty}|f(x)| d x<\infty$. If $p(x) \equiv 1$ on $I$, then $N\left(x_{0}, 4(n-1) / H\right)<n$. If $p(x) \equiv x$ on I, then $N\left(x_{0}, x_{0} \exp [4(n-1) / H]\right)<n . \quad$ If $p(x) \equiv x^{2}$ on $I$, then $N\left(x_{0}, \infty\right)<$ $\left(H / 4 x_{0}\right)+1, x_{0}>0$, hence the equation is nonoscillatory on $I$.

Proof. If $p(x) \equiv 1$ on $I$, then from equation (16) $s=(a+b) / 2$ and the maximum value of $|g(x, s)|=(b-a) / 4$. From inequality (7),

$$
1<\max |g(x, s)| \int_{a_{j}}^{a_{j+1}}|f(x)| d x, \quad j=1,2, \cdots, n-1
$$

so that

$$
n-1<\frac{a_{n}}{4} \int_{x_{0}}^{\infty}|f(x)| d x=\frac{H a_{n}}{4},
$$

and $a_{n}>4(n-1) / H$. The results for $p(x) \equiv x$ and $p(x) \equiv x^{2}$ can be obtained in a similar fashion.

4. The equation $y^{\prime \prime \prime}+f(x) y=0$. The differential system $y^{\prime \prime \prime}=0$, $y(a)=y(b)=y(c)=0, a<b<c$, is incompatible, so that the Green's function for this system exists and has the explicit form

(17) $g(x, s)$

$$
=\left\{\begin{array}{l}
\frac{1}{2} \frac{(c-s)^{2}}{(c-a)(c-b)}(x-a)(x-b)=g_{11}, b<s<c, a \leq x \leq s \\
g_{11}-\frac{1}{2}(x-s)^{2}=g_{12}, b<s<c, s \leq x \leq c \\
g_{11}-\frac{1}{2} \frac{(b-s)^{2}}{(c-b)(b-a)}(x-a)(x-c)=g_{21}, a<s<b, a \leq x \leq s \\
g_{21}-\frac{1}{2}(x-s)^{2}=g_{22}, a<s<b, s \leq x \leq c \\
\frac{1}{2} \frac{c-b}{c-a}(x-a)(x-b)=g_{31}, s=b, a \leq x \leq s \\
g_{31}-\frac{1}{2}(x-s)^{2}=g_{32}, s=b, s \leq x \leq c .
\end{array}\right.
$$


An upper bound for $|g(x, s)|$ on $a \leq x \leq c, a<s<c$ can be obtained when $a \geq 0$ by considering each of the expressions $g_{i j}$ above. It is easily found that

$$
\left|g_{11}\right|<\frac{c^{2}}{2},\left|g_{12}\right|<c^{2},\left|g_{31}\right|<\frac{c^{2}}{2},\left|g_{32}\right|<c^{2} .
$$

The expression for $g_{22}(x, s)$ can be written as

$$
g_{22}=\frac{1}{2} \frac{(s-a)^{2}}{(c-a)(b-a)}(x-b)(c-x),
$$

whence $\left|g_{22}\right|<c^{2} / 2$, and $\left|g_{21}\right| \leq\left|g_{22}\right|+(1 / 2)(x-s)^{2}<c^{2}$. Thus in each case $\left|g_{i j}\right|<c^{2}$, so

$$
|g(x, s)|<c^{2} \text { for } a \leq x \leq c, a<s<c .
$$

Assume $f(x)$ is continuous on $I$. If $y(x)$ is a nontrivial solution of equation (2) on the interval $a \leq x \leq c$ for which $y(a)=y(b)=y(c)=0$, $0 \leq x_{0} \leq a<b<c$, then

$$
y(x)=\int_{a}^{c} g(x, s) y(s) f(s) d s,
$$

and as in $\S 1$,

$$
1<\int_{a}^{c}|g(x, s)||f(s)| d s .
$$

Using inequality (18),

$$
1<c^{2} \int_{a}^{c}|f(x)| d x
$$

Theorem 5. Suppose $f(x)$ is continuous on $I: x_{0} \leq x<\infty, x_{0} \geq 0$, and $\int_{x_{0}}^{\infty}|f(x)| d x=N$. If $a_{1}<a_{2}<\cdots<a_{n}$ are $n$ consecutive zero of $a$ solution of $y^{\prime \prime \prime}+f(x) y=0, a_{1} \geq x_{0}$, then

$$
a_{n}>\sqrt{\left[(n-1)-\left(1+(-1)^{n}\right) / 2\right] / 2 N}, n \geq 3 .
$$

Proof. From inequality (19),

$$
1<a_{j+2}^{2} \int_{a_{j}}^{a_{j+2}}|f(x)| d x, \quad j=1,2, \cdots, n-2 .
$$

Let $n=2 m$. Then adding the inequalities in (21) for $j=2,4, \cdots, 2 m-2$,

$$
m-1<a_{2 m}^{2} \int_{a_{2}}^{a_{2 m}}|f(x)| d x<a_{2 m}^{2} N .
$$

Therefore $a_{2 m}=a_{n}>\sqrt{(n-2) / 2 N}$. If $n=2 m+1$, then adding ine- 
qualities in (21) for $j=1,3, \cdots, 2 m-1, m<a_{2 m+1}^{2} N$, so $a_{2 m+1}=a_{n}>$ $\bar{V}(n-1) / 2 N$. Combining these two cases the inequality (20) results.

Note 2. Adding the $n-2$ inequalities in (21),

$$
n-2<2 a_{n}^{2} \int_{x_{0}}^{a_{n}}|f(x)| d x .
$$

In the case when $N=\int_{x_{0}}^{\infty}|f(x)| d x=\infty$, this last inequality still yields lower bounds for the zeros $a_{n}$. For example, if $f(x)=\sqrt{x}+i$ and $x_{0}=0$, then

$$
n-2<\frac{4}{3} a_{n}^{2}\left[\left(a_{n}+1\right)^{3 / 2}-1\right], n \geq 3,
$$

and the positive root of

$$
x^{7}+3 x^{6}+3 x^{5}-\frac{3}{2}(n-2) x^{2}-\frac{9}{16}(n-2)^{2}=0
$$

is a lower bound for $a_{n}$.

5. Higher order equations. The methods employed in deriving inequalities (14) $(\sigma=0)$ and (20) can be applied to the $k$ th order differential equation

$$
y^{(k)}+f(x) y=0,
$$

where $f(x)$ is continuous and complex-valued on $I$. For suppose $a_{1}<$ $x_{2}<\cdots<a_{n}$ are $n$ consecutive zeros of a solution of equation (22), $x_{1} \geq x_{0} \geq 0, n=k q+r \geq k$. Then

$$
1<\int_{a_{j}}^{a_{j+k-1}}|g(x, s)||f(x)| d x, \quad j=1,2, \cdots, n-k+1,
$$

where $g(x, s)$ is the Green's function for the system.

$$
y^{(k)}=0, y\left(a_{j}\right)=y\left(a_{j+1}\right)=\cdots=y\left(a_{j+k-1}\right)=0 .
$$

Suppose a bound can be found for $|g(x, s)|$ on the interval $a_{j} \leq x \leq a_{j+k-1}$ which is a monotone function, say $B\left(a_{j+k-1}\right)$, of $a_{j+k-1}$. Then

$$
q<B\left(a_{n}\right) \int_{x_{0}}^{a_{n}}|f(x)| d x \leq B\left(a_{n}\right) N,
$$

where $N=\int_{x_{0}}^{\infty}|f(x)| d x \leq \infty$. In particular if we conjecture ${ }^{2} B\left(a_{n}\right)<a_{n}^{k-1}$, as is the case for $k=2,3$, then

$$
a_{n}>\sqrt[k-1]{\frac{n-(k-1)}{k N}} .
$$

2 This conjecture has been verified for $n<6$. 


\section{REFERENCES}

1. J. H. Barrett, Second order complex differential equations with a real independent variable, Pacific J. Math. 8 (1958), 187-200.

2. J. H. Barrett, W. J. Coles, D. V. V. Wend and R. W. Hunt, Complex differential equations I, O. O. R. Technical Report, University of Utah (1958), 1-39.

3. P. R. Beesack, Nonoscillation and disconjugacy in the complex domain, Trans. Amer. Math. Soc. 81 (1956), 211-242.

4. E. Hille, Non-oscillation theorems, Trans. Amer. Math. Soc. 64 (1948), 234-252.

5. W. Leighton, On self-adjoint differential equations of second order, J. Lond. Math. Soc. 27 (1952), 37-47.

6. R. A. Moore, The behavior of solutions of a linear differential equation of the second order, Pacific J. Math. 5 (1955), 125-145.

7. Z. Nehari, On the zeros of solutions of second-order linear differential equations, Amer. J. Math. 76 (1954), 689-697.

8. R. L. Potter, On self adjoint differential equations of second order, Pacific J. Math. 3 (1953), 467-491.

UNIVERSITY OF UTAH 


\section{PACIFIC JOURNAL OF MATHEMATICS}

\section{EDITORS}

David Gilbarg

Stanford University

Stanford, California

F. H. Brownell

University of Washington

Seattle 5, Washington
A. L. Whiteman

University of Southern Californıa Los Angeles 7, California

L. J. PaIge

University of California

Los Angeles 24, California

\section{ASSOCIATE EDITORS}
E. F. BECKENBACH
T. M. CHERRY
D. DERRY

E. HEWITT
A. HORN
L. NACHBIN
M. OHTSUKA

H. L. ROYDEN

M. M. SCHIFFER
E. SPANIER

E. G. STRAUS

F. WOLF

\section{SUPPORTING INSTITUTIONS}

\author{
UNIVERSITY OF BRITISH COLUMBIA \\ CALIFORNIA INSTITUTE OF TECHNOLOGY \\ UNIVERSITY OF CALIFORNIA \\ MONTANA STATE UNIVERSITY \\ UNIVERSITY OF NEVADA \\ NEW MEXICO STATE UNIVERSITY \\ OREGON STATE COLLEGE \\ UNIVERSITY OF OREGON \\ OSAKA UNIVERSITY \\ UNIVERSITY OF SOUTHERN CALIFORNIA
}

\author{
STANFORD UNIVERSITY \\ UNIVERSITY OF TOKYO \\ UNIVERSITY OF UTAH \\ WASHINGTON STATE COLLEGE \\ UNIVERSITY OF WASHINGTON \\ AMERICAN MATHEMATICAL SOCIETY \\ CALIFORNIA RESEARCH CORPORATION \\ HUGHES AIRCRAFT COMPANY \\ SPACE TECHNOLOGY LABORATORIES \\ NAVAL ORDNANCE TEST STATION
}

Mathematical papers intended for publication in the Pacific Journal of Mathematics should be typewritten (double spaced), and the author should keep a complete copy. Manuscripts may be sent to any one of the four editors. All other communications to the editors should be addressed to the managing editor, L. J. Paige at the University of California, Los Angeles 24, California.

50 reprints per author of each article are furnished free of charge; additional copies may be obtained at cost in multiples of 50 .

The Pacific Journal of Mathematics is published quarterly, in March, June, September, and December. The price per volume (4 numbers) is $\$ 12.00$; single issues, $\$ 3.50$. Back numbers are available. Special price to individual faculty members of supporting institutions and to individual members of the American Mathematical Society: $\$ 4.00$ per volume; single issues, $\$ 1.25$.

Subscriptions, orders for back numbers, and changes of address should be sent to Pacific Journal of Mathematics, 2120 Oxford Street, Berkeley 4, California.

Printed at Kokusai Bunken Insatsusha (International Academic Printing Co., Ltd.), No. 6, 2-chome, Fujimi-cho, Chiyoda-ku, Tokyo, Japan.

PUBLISHED BY PACIFIC JOURNAL OF MATHEMATICS, A NON-PROFIT CORPORATION

The Supporting Institutions listed above contribute to the cost of publication of this Journal, but they are not owners or publishers and have no responsibility for its content or policies. 


\section{Pacific Journal of Mathematics}

\section{Vol. 10, No. $2 \quad$ October, 1960}

Maynard G. Arsove, The Paley-Wiener theorem in metric linear spaces ........

Robert (Yisrael) John Aumann, Acceptable points in games of perfect

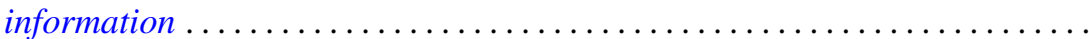

A. V. Balakrishnan, Fractional powers of closed operators and the semigroups generated by them ... . . . . . . . . . . . . . . . . . . . . . . . . . . . . 419

Dallas O. Banks, Bounds for the eigenvalues of some vibrating systems . . . . . 439

Billy Joe Boyer, On the summability of derived Fourier series . . . . . . . . . . . 475

Robert Breusch, An elementary proof of the prime number theorem with

remainder term ...................................

Edward David Callender, Jr., Hölder continuity of $n$-dimensional

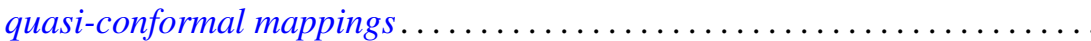

L. Carlitz, Note on Alder's polynomials ......................... 517

P. H. Doyle, III, Unions of cell pairs in $E^{3} \ldots \ldots \ldots \ldots \ldots \ldots \ldots \ldots \ldots \ldots \ldots \ldots . \ldots 21$

James Eells, Jr., A class of smooth bundles over a manifold . . . . . . . . . . . . 525

Shaul Foguel, Computations of the multiplicity function . . . . . . . . . . . . . . 539

James G. Glimm and Richard Vincent Kadison, Unitary operators in

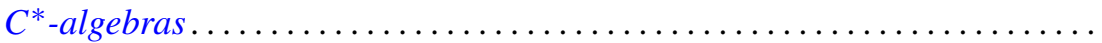

Hugh Gordon, Measure defined by abstract $L_{p}$ spaces . . . . . . . . . . . 557

Robert Clarke James, Separable conjugate spaces ....................

William Elliott Jenner, On non-associative algebras associated with bilinear forms

Harold H. Johnson, Terminating prolongation procedures

John W. Milnor and Edwin Spanier, Two remarks on fiber homotopy type .

Donald Alan Norton, A note on associativity . .

Ronald John Nunke, On the extensions of a torsion module.

Joseph J. Rotman, Mixed modules over valuations rings . . . . .

A. Sade, Théorie des systèmes demosiens de groupoï des . .

Wolfgang M. Schmidt, On normal numbers . .

661

Berthold Schweizer, Abe Sklar and Edward Oakley Thorp, The metrization of

statistical metric spaces

John P. Shanahan, On uniqueness questions for hyperbolic differential

equations

A. H. Stone, Sequences of coverings

Edward Oakley Thorp, Projections onto the subspace of compact operators

L. Bruce Treybig, Concerning certain locally peripherally separable spaces

Milo Wesley Weaver, On the commutativity of a correspondence and a

permutation

David Van Vranken Wend, On the zeros of solutions of some linear complex

differential equations. 